\title{
Sindrome Nefrótico Idiopático III Parte: Tratamiento y Pronóstico
}

DRES.: F, PUGA C. *, E. RODRIGUEZ s. *, H. ROSENBERG **.

Los lentos progresos logrados en el mejor entendimiento del Sindrome Nefrótico Idiopático tanto en etiología, patología y patogenia no se reflejan en un mejoramiento de los medios de tratamiento a disposición del clínico.

El gran beneficio logrado por los antibióticos y quimioterápicos introducidos en las décadas del 30 al 50 redujeron en forma acentuada la alta mortalidad producida por infecciones, generalmente graves, que ocurren en estos enfermos $(7,14)$.

En 1955 se introduce la corticoterapia, lográndose una buena respuesta inmediata en lo que se refiere a la crisis nefrótica misma, sin que haya demostrado beneficio en el pronóstico a largo plazo de la enfermedad. Su aplicación, universalmente difundida obliga, hasta cierto punto, a su uso en todo paciente con sindrome nefrótico, con algunas excepciones bien calificadas, aunque existen muchas reservas mentales en cuanto a un efecto curativo, reconociéndose sí sus beneficios inmediatos $(10,11,12,17)$.

Los esquemas terapéuticos han variado de aquellos que duraban dos años o más, a plazos más cortos, de 3 a 6 meses, ya sea con tratamientos continuos o intermitentes, con el fin de proteger la función de las Suprarrenales, sin apreciarse diferencias en sus resultados sobre el riñón $(1,2,3)$.

Luego aparecen los diuréticos tiazídicos, antialdosterónicos (4) y últimamente otros más potentes como el ácido etacrínico y furosemida, todos con malos resultados $(8,9)$. Los citotóxicos $\mathrm{e}$ inmunosupresores han revelado ser beneficiosos en algunos de estos pacientes, particularmentc

\footnotetext{
- Deparlamento de Netrourulagia. Hospilal Luis Calvo Mackerna.

* Departamento de Anatomía Pałológica. Hospital Clínico U. Católica.
}

cuando se trata de niños con recaídas frecuentes, pero su aplicación tiene riesgos y obliga a uni buena selección antes de su indicación $(5,6,15)$.

Por último, el uso de heparina y fibrinolíticos como el dipiridamol están en etapa de investigación con resultados aún dudosos.

Nuestra experiencia terapéutica ha consistido en el uso de corticoides, antibióticos, diuréticos $\mathrm{e}$ inmunosupresores cuyos resultados damos a conocer.

Material y MÉTodo. De 90 pacientes con Sindrome Nefrótico Idiopático, 83 recibieron tratamiento con corticoides (Prednisona) siguiendo 2 esquemas:

a) $2 \mathrm{mg} / \mathrm{kg}$ al día por 3 meses y luego reducción progresiva de acuerdo a la presencia o ausencia de proteinuria, prolongándose el tratamiento por un plazo de hasta 2 años (1961 a 1968).

b) $2 \mathrm{mg} / \mathrm{kg}$ de Prednisona al día por 30 días y luego igual dosis 4 días a la semaná por un período de dos meses hasta completar un tratamiento total de 3 meses. (1968 adelante).

Los controles se realizaron cada 15 a 30 días en los períodos de actividad y cada 2 a 4 meses en los períodos de remisión y hasta 5 años y más de duración de esta remisión.

Antibióticos, de preferencia la Penicilina, se usaron cuando existió una infección activa. Estas han sido 5 Erisepelas, 7 Neumonias, 3 Peritonitis primarias y 1 Meningitis purulenta, mencionando solamente las infecciones piógenas graves.

Tanto la varicela, sarampión, herpangina y parotiditis fueron frecuentes, en los primeros años, 
cuando las hospitalizaciones de nuestros pacientes eran muy prolongadas y no influyeron en el curso de la enfermedad.

En varios pacientes se usó hidrociorotiazida (8), triamterene, quinecazona (8), furosemida y ácido etacrínico. Cuando la proteinemia es inferior a $40 \mathrm{gr} / \mathrm{mil}$ habitualnente la respuesta de los diuréticos es nula, pero su respuesta se ve favorecida con el uso de plasma a razón de $10 \mathrm{ml} / \mathrm{kg}$ puesto en forma rápida (60 a 80 gotas por min.), seguido luego del diurético por vía endovenosa.

En 25 pacientes se usó ciclofosfamida por períodos de 3 meses en dosis de $5 \mathrm{mg} / \mathrm{kg}$ diario con $1 \mathrm{mg}$. de pređinisona por kilo mientras duró el tratamiento. Estos pacientes presentaban recaídas frecuentes o eran resistentes a la terapia esteroidal. Además un caso recibió Clorambucil y 12 Azathioprine.

En 7 pacientes con sindrome nefrótico idiopático no se uś́ tratamiento con corticoides. Uno de ellos tenía lesiones mínimas y consultó en remisión y no ha vuelto a recaer; otros 2 pacientes con glomerulonefritis proliferativa mesangial por la misma razón y otros 4 niños por el hallazgo histológico que no hizo recomendable el uso de drogas.

DDel total, 9 fallecieron y en 7 de ellos se hizo autopsia.

Resultados. La respuesta de $\operatorname{los} 83$ pacientes que recibieron tratamiento con prednisona purede verse en el Gráfico 1. Como era de esperar, 47 de los 54 niños con lessiones mínimas que tuvieron tratamiento fueron sensibles, pero de éstos 23 se hicieron dependientes a corto plazo de suspendido el tratamiento. En 7 de éstos se usó ciclofosfamida con buen resultado. Sólo 7 fueron resistentes a la prednisona. De estos resistentes 5 recibieron tratamiento con ciclofosfamida con mal resultado. Los resultados con inmunosupresores se analizarán en un trabajo aparte.

SINUROME NEFROTICO IDIOPATICO

RELACION RESPUESTA A PREDNHSONA Y LESION

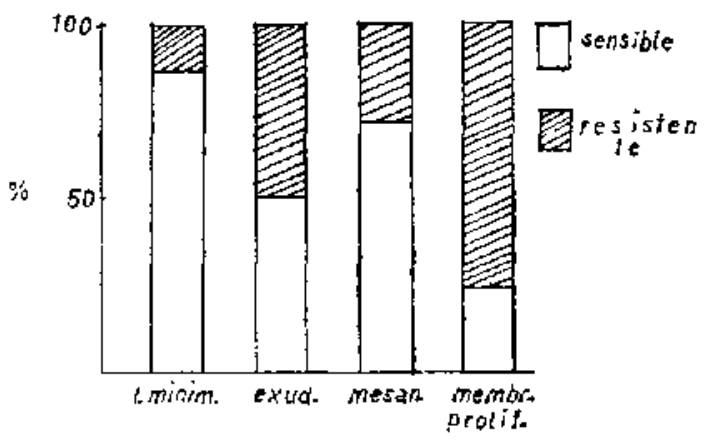

Algo similar sucede con los niños con lesiones de Glomerulonefritis difusa proliferativa mesangial, aunque en este grupo el porcentaje de beneficiados es menor y hay 10 sensibles y 4 resistentes. Gráfico 1. Uno de estos resistentes se trató con ciclofosfamida con mal resultado y 2 niños dependientes al tratamiento mejoraron definitivamente.

En los pacientes con glomerulonefritis difusa proliferativa y exudativa (G. N. D. P. E.), Glomerulonefritis Membrano Proliferativa (G. N. M. P.), Glomerulonefritis Focal (G. N. F.), y Glomerulopatía Membranosa (G. M.) los resultados frente al corticoides fueron en general pobres, to que también se vio con la ciclofosfamida.

Ła remisión con el tratamiento esteroidal fue rápida en la mayoría de los pacientes $y$ en especial en los con lesiones mínimas. De los 54 con lesiones mínimas tratados, 47 remitieron antes de los 3 meses de iniciado el tratamiento, de los cuales 43 ya habían remitido antes del mes. Algo semejante ocurrió con la G. N. D. P. M. De los 7 resistentes con lesiones mínimas sólo 4 lo fueron desde el primer tratamiento y los otros 3 se hicieron resistentes en tratamientos posteriores. Vale la pena destacar que 2 de éstos últimos se hicieron resistentes después de padecer una hepatitis infecciosa.

Los otros grupos de lesiones fueron más lentos para responder o eran resistentes.

La curación de la remisión, después de suspendido el tratamiento, tanto en el tratamiento corto como largo es semejante y tanto los pacientes con L. M. y G. N. D. P. M. tienen cortos períodos de remisión y por ende, recaídas frecuentes.

Ś́lo 10 de los $54(18,5 \%)$ pacientes con Jesiones mínimas no hicieron más de una crisis nefrótica; Ios demás recayeron, en su mayoria, antes de los 6 meses de suspendido el tratamiento. En aquellos con G. N. D. P. M. hay 3 que sólo tuvieron un episodio clínico y en los otros hubo, en general, recaidas precoces. La G. N. D. P. E. son de remisión más lenta, pero ésta es definitiva.

En la G. N. M. P. los corticoides no fueron eficaces y en cambio pueden provocar hipertensión y aumento de la uremia, por lo que su uso está contraindicado.

La Ciclofosfamida o Cloranbucil deben usarse en los casos sensibles a corticoides con recaídas frecucntes o tal vez en aquellos casos con lesiones inflamatorias agudas activa siempre que no exista ya insuficiencia renal avanzada, por el riesgo que esta droga significa para estos pacientes.

Las rápidas remisiones con el tratamiento $\mathrm{y}$ las recaídas a corto plazo hacen que estos pacientes tengan numerosos episodios nefróticos que requieren repetir el tratamiento, en lo posible ambulatorio, debiendo reservarse la hospitalización 
solamente para aquelios estudios especiales (Biopsia) o bien por las condiciones del niño (infecciones, edema doloroso muy acentuado, tratamiento inicial con Ciclofosfamida, razones sociales).

El sindrome nefrótico está constituido por numerosas y distintas enfermedades como lo indica su nombre, y muchas de éstas son enfermedades autolimitadas en el tiempo. Los antibióticos indudablemente han sido muy favorables en el tratamiento de infecciones graves como las que presentan estos pacientes y el corticoide ha acortado los períodos críticos de la enfernedad provocados por el intenso edema, invalidante en algunas ocasiones. Sin embargo, el corticoide no tendría un efecto curativo en ninguno de los tipos hasta ahora mencionados de Sindrome Nefrótico Idiopático y su uso no es inocuo. Entre sus riesgos la curva estatural se detiene durante el tratamiento estcroidal, pero ésta se recupera rápidamente una vez suspendido, alcanzando los niños una estatura normaI a los 2 a 3 años de estar en remisión.

La aparición de cara de luna la hemos observado con cualquier esquema terapéutico (continuo, discontinuo o intermitente) y con la excepción de un fallecido a consecuencia de la suspensión de un tratamiento intermitente al cumplir el plazo programado, no hemos observado otros casos con insuficiencia suprarrenal.

El réginen sin sal lo indicamos solamente durante el estado adematoso y mientras esté recibiendo prednisona.

Los resultados con el uso de citostáticos e inmunosuprosores será analizado en otra publicación.

Por último, y no por eso menos importante, los padres y el niño deben ser informados sobre las características de la enfermedad. Las frecuentes recaídss que requieren nuevos tratamientos; los riesgos de infecciones graves, muchas veces poco aparentes; la necesidad de realizar exámenes (Biopsias) que requerirán hospitalizaciones cortas. Sólo el edema es una razón de limitación en las actividades normales para cualquier niño. Evitar el abuso en Ias tomas de muestras innecesarias $y$ muchas veces doloroses. Ofrecerles una imagen pronóstica objetiva, habitualmente favorable y en cuanto fuera posible que sea un mismo médico que intervenga en el seguimiento del enfermo.

Es nuestra impresión que la mayoría de los sindromes nefróticos idiopáticos $y$, en especial aquellos con lesiones mínimas o Glomerulonefritis Proliferativa Mesangial son enfermedades autojimitadas observándose una remisión definitiva alrededor de los cuatro años.

Es indispensable continuar lá búsqueda de ele. mentos clínicos y de laboratorio que nos permitan hacer un pronóstico y predecir el momento en el que el niño entra en una remisión definitiva.

Por el momento, los medios a nuestro alcan- ce son limitados, pero pueden mencionarse algunos:

La edad de comienzo y los caracteres clínicos de sindrome nefrótico puro o impuro; la sensibilidad a la respuesta terapéutica esteroidal y el tiempo de observación son de gran apoyo, pero muy falibles. La normalización de la curva electroforética de las proteínas del plasma y el colesterol plasmático en niveles normales reflejan una remisión, pero no una curación.

En nuestra experiencia nos ha resultado muy fiel la normalización de las inmunoglobulinas plasmáticas y en especial la IgG e IgM que persisten alteradas en los casos de mal pronóstico, o en las remisiones con posibles recaídas, alcanzando niveles normales solamente en aquellos pacientes que no van a recaer.

No tenemos experiencia en la determinación de la selectividad en la proteinuria, pero ella tam bién sería un apoyo, particularmente en niños, pues en éstos existió mejor correlación entre las lesiones histológicas y el tipo de proteinuria (16).

La Biopsia renal es indudablemente un excelente medio para fijat un pronóstico y en especial si esta se repite. Sin embargo, aún con aquellas lesiones "benignas" nos ofrece un pronóstico a Iargo plazo, pero no responde a la necesidad inmediata de curación. Parece que la Microscopía electrónica puede abrir nuevas expectativas.

Sin duda, el ponderado análisis del conjunto de todos estos datos y antecedentes clínicos $\mathrm{e}$ histológicos pueden permitir al médico una información adecuada sobre el pronóstico inmediato y a largo plazo de su paciente.

\section{RESUMEN}

Se analizan los diversos medios terapéuticos aplicados al Sindrome Nefrótico Idiopático del niño. En 83 pacientes se usó prednisona a dosis de $2 \mathrm{mg} / \mathrm{k} / \mathrm{d}$ ia, siguiendo dos esquemas; uno prolongado hasta de dos años y otro de 3 meses, no encontrándose diferencia significativa en sus resultados.

La respuesta fue favorable en los enfermos con lesiones minimas y Glomérulonefritis proliferativa mesangial, haciéndose frecuentemente dependiente al corticoide, pero en general con buena respuesta a los citotóxicos.

Los enfermos con Glomérulonefritis difusa proliferativa exudativa, glomérulonefritis membrano-proliferativa, glomérulonefritis focal y glomerulopatia membranosa son en su mayoría resistentes al esteroide. Se destaca los riesgos y complicaciones del uso de corticoides en niños y la información necesaria que deben recibir los padres de estos enfermos. 


\section{SUMMARY}

Therapeutic elements in the treatment of Idiophatic Nephrotic Syndrome in children are analysed: Antibiotics; Diuretics; Prednisone, Cytotoxics and heparine.

Prednisone was used in 83 patients following two schemes: one long up two years and one short for 3 months.

Minimal lesions and mesangial proliferation haye good results, but often become dependent to sterojd. Most of them recover to cytotoxics drugs.

Parents and pacients must be informed of the natural history of the disease and bed rest used only as an exception.

Some clinical and laboratory tests are considered in the prognosis.

\section{REFERENCIAS}

1.- Soyles J. D. "Alternate-Day steroid therapy for Nephrotic children". J. Am. Med. Ass. 192/3 (225230), 1965.

3.- Soyka L. F. "The Nephrotic Syndrome. Current concepts in diagnosis and therapy advantages of alternateday steroid regimen". Clinic. Pediat. 6: 77, Feb. 1967.

3.--Soyka L. F. "Treatment of the Nephrotic Syndrome in childhood. Use of an alternate-day prednisone regimen". Am. J. Dis. Child. 113: 693, Jun. 1967.

4.-Ruff H. "The effect of Hydroclorothiazide assosciated with Antialdosterone drougs in the trcatment of Nephrotic Syndrome oedema". Progr. Med. (Napoli), 19/17 (521-529), 1963.

5.- Northway J. D., West C. D. "Succesful therapy of trimethadione nephrosis with prednisone and cyclophosfamide". J. of Pediat. 71: 259, 1967.
6.- Grufe W. E. "Cytotoxic drougs in steroit-resistant renal disease alkalative and antimetabolic agents in the treatment of Nephrotic Syndrome, Lupus nephritis, Chronic Glomerulonephritis, and Purpure nephritis in children". Am. Jour Dis. Child, 112: 448, Nov, 1966.

7.-Kolm, J. "Nephrotic Syndrome in children". Am. Jour. Dis Child. 100: 373, 1960.

8.- Piga F.. Ugarie J. M., Frías J. "Fusión rápida del edema en el Sinđtome Neftótico". Rev. Chil. Ped. 36: 234,1965 .

9.-Hutelum J. L. "Expansión del volumen plasmático después de la inyección de plasma". I. Lab. and Clin. Med, 56: 734, 1960.

10.--Saxena K. M., Graeford J. D. "Treatment of the Nephrosis". The New Eng. J, Med. 272: 522, 1965.

11.-Brown R. B. "Studies in Nephrotic Synk1rome. I survival of 135 children with nephrotic syndrome treatted with adrenal steroid". Proc. Mayo Clin. $40 / 5,(384-390), 1965$.

12.- Ameil G. L. "Steroid therapy in childhood nephrosis. Lowg term assessement". The Lancet. 2, 819$82 \mathrm{I}, 1966$.

13.- Cornfeld D. "Nephrosis: A long term study of children treated with corticosteroid". J. of Pediat. 68: 507, April, 1966.

14.-Hooff $D$. "The natural history of Idiopathic Nephrotic Syndrome in childhood". Ann Pediat. 207: 1, 1966.

15.-Adams D. A. "Azathioprine treatment of inmunologicat renal disease". Jama 199: 459, 1967.

16,- Black D. A. K. "Renal disease". Blackwell Scientific Publ. Oxford, 1966. 